\title{
Development and application of a closed-loop medication administration system in University of Hongkong-Shenzhen Hospital ${ }^{\dagger}$
}

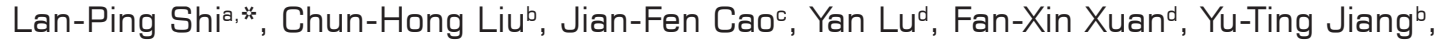
Jin-Yang Zhouc

aNursing Department, University of Hong Kong-Shenzhen Hospital, Shenzhen, Guangdong 518053, China

${ }^{b}$ Endocrine Department, University of Hong Kong-Shenzhen Hospital, Shenzhen, Guangdong 518053, China

cMedicine Department, University of Hong Kong-Shenzhen Hospital, Shenzhen, Guangdong 518053, China

'Hemodialysis Department, University of Hong Kong-Shenzhen Hospital, Shenzhen, Guangdong 518053, China

Received: 20 September 2017; Accepted: 23 November 2017; Published: 20 June 2018

Abstract: Objective: This study aimed to develop and apply a closed-loop medication administration system in a hospital in order to reduce medication administration errors (MAEs).

Methods: The study was implemented in four pilot general wards. We used a before-and-after design to collect oral medication administration times before and after the implementation of the closed-loop medication administration system, evaluated MAE alert logs after the intervention, and conducted a survey of the nurses' satisfaction with the system in the pilot wards.

Results: (a) Nursing time of oral medication administration: before the adoption of the closed-loop medication administration system, the average nursing time was $31.56 \pm 10.88$ minutes $(n=78)$; after the adoption of the system, the time was $18.74 \pm 5.60$ minutes $(n=54)$. Independent sample $t$-tests showed a significant difference between two groups $(t=8.85, P<0.00)$. (b) Degree of nurses' satisfaction with the closed-loop medication administration system: $60.00 \%(n=42)$ of nurses considered the system to be helpful for their work and nearly half of the nurses $(47.14 \%, n=33$ ) believed that the system could facilitate clinical work and reduce workload; $51.43 \%(n=36)$ believed that the system could reduce checking time and enhance work efficiency; $82.86 \%(n=58)$ believed that the system was helpful in improving checking accuracy to reduce MAEs and ensure patient safety. More than $60 \%$ of the nurses considered the system to be a method that could help to track MAEs to improve nursing quality. (c) The MAE alert logs during observation period: it revealed only 27 alerts from the repeated scans of 3,428 instances of medication administration.

Conclusions: The nurses were satisfied with the closed-loop medication administration system because it improved their work efficiency and reduced their workload. The current investigation was limited by time; therefore, further research is needed to more closely examine the relationship between the system and MAEs.

Keywords: medication error • medication administration • closed-loop system • computerized physician order entry system

(c) Shanxi Medical Periodical Press.

\section{Introduction}

A medication administration error (MAE) is defined as a medication error occurring during the bedside

\footnotetext{
${ }^{\dagger}$ This project was supported by Health and Family Planning Commission of Shenzhen Municipality, Scientific Research Foundation (2015), No. 31 (No. 201504004)
}

*E-mail:shilp@hku-szh.org administration of a medication. ${ }^{1}$ Studies have shown that MAEs cannot be prevented by any combination of computerized physician order entry (CPOE), electronic health record (EHR), clinical decision support system, or an automated dispensing system but can only be mitigated by a closed-loop medication administration system. ${ }^{2-5}$ The closed-loop medication administration system includes automated identification technology 
such as radio-frequency identification (RFID) and/or barcodes. ${ }^{6,7}$ It is used to verify the so-called "five rights" (5R; right patient, right drug, right dose, right route, and right time) of medication administration at the bedside by cross-checking patients' identification, prescription information, and dispensed drugs using a hand-held pointof-care device with RFID and/or a barcode reader. ${ }^{8,9}$

In 1999, the Institute of Medicine ${ }^{10}$ reported that medical errors result in 44,000-98,000 preventable deaths and more than 1,000,000 injuries each year in US hospitals. Medication errors can occur at any stage of the medication process, including prescribing, transcribing, dispensing, and administration. ${ }^{11}$

Previous research has indicated that closed-loop medication administration systems reduce non-intravenous MAEs to $39 \%$ and also reduce the administration of the wrong dose as well as omission errors. ${ }^{12}$ However, personal digital assistant (PDA) or hand-held point-of-care devices have been adopted in only $27.19 \%$ hospitals in China due to difficulties with application, high implementation costs, and maintenance fees. ${ }^{13}$

The present study was conducted at the University of Hongkong-Shenzhen Hospital (HKU-SZH) from August 1, 2016, to December 31, 2016. The hospital is a general teaching hospital affiliated with the University of Hongkong and owned by Shenzhen government. It opened in 2012 and has 2,000 beds and an out-patient capacity of $8,000-10,000$ patients. The HKU-SZH has set up a wireless environment since its beginning and has adopted a clinical information system including CPOE, EHR, a clinical decision support system, and picture archiving and communication systems.

In 2015, the HKU-SZH reported 105 medication errors out of 515 incidents (20.39\%). The aim of this study was to determine whether the development and implementation of a closed-loop medication administration system could reduce MAEs in the hospital.

\section{Methods}

Prior to commencing the study, a literature review was performed in order to analyze the workflow of medication administration in clinical settings and a diverse project team was set up, which consisted of an information technology (IT) manager, IT engineers, pharmacists, doctors, head nurses, and registered nurses (RNs). The project plan had four steps: (a) preparation period: develop the system and finish PDA procurement; (b) pilot study: apply the system in pilot wards and modify the system accordingly; (c) nurse training and application of the system to whole hospital; and (d) data collection and analysis and summarization of the findings in a paper.

\subsection{System development and technology support}

\subsubsection{System development}

The closed-loop medication administration system was developed using Microsoft Visual Studio Net 2005. The system adopted an object-oriented design, programmed with Microsoft Foundation Classes; repacked the controls; and perfected the system function. The client and server system consisted of three layers of logically separated frameworks: the user interface (UI) layer, the Buss' rule layer, and the data access layer.

The closed-loop medication administration system requires technical support to obtain clinical information, which mainly includes the following: (a) in-patients wear barcoded wristbands, from which patient information can be read; (b) the closed-loop medication system is based on a CPOE system in order to achieve intelligent and structured prescription records; (c) an automatic drug dispensing system is applied to the pharmacy in order to integrate drug and patient information; and (d) nurses use PDAs and mobile workstations to identify patients, drug and prescription information, and match information and automatically obtain execution confirmation.

\subsection{Workflow}

In the traditional paper-driven process of medication administration, two nurses manually double-check the medication information including name, dose, time, route of medication, and the patient's identity before the medication is administered. ${ }^{14}$

With the closed-loop medication administration system, the process of medication administration requires nurses to scan the barcodes on the patient's wristband as well as the barcodes on the medication before it is administered. ${ }^{15,16}$ If the dose being scanned corresponds with the approved medication order of a pharmacist and the patient is due to receive this medication, the administration is automatically documented in real time. However, if the medication does not correspond to a valid order, the system issues a warning.

Since the closed-loop medication administration system provides an additional layer of safety with the real-time scanning of barcodes, ${ }^{17}$ we changed the process of medication administration after application of the system from requiring two nurses at the bedside to perform the double-check to the following two methods: a single nurse (a) manually checked as routine at the bedside and (b) scanned the barcodes on both patient's wristband and the medication before medication administration. 


\subsection{Study design}

The study was implemented in four pilot general wards. We used a before-and-after design and collected data on oral medication administration times before and after the system implementation. We also evaluated the MAE alert logs after the implementation and surveyed the nurses' satisfaction with the system.

\subsection{Survey tool}

Self-reported satisfaction questionnaires were used for the survey. A brainstorming meeting of the clinical head nurses was used to design the first draft of the questionnaire. A pretest of the questionnaire was conducted in the pilot wards; based on the results of the pretest, we further modified the questionnaire to develop the formal version. The formal questionnaire contained questions from five perspectives, including the quality of nursing, patient safety, nurse workload, work efficiency, and nurses' views of the PDA. Each question was answered according to five degrees: strongly disagree, disagree, neutral, agree, and strongly agree.

\subsection{Data collection}

Four nursing students observed and recorded the oral medication administration times in the four pilot wards respectively before and after intervention (December 12-16, 2016, and January 2-6, 2017). The satisfaction questionnaire survey was conducted in the four pilot wards on January 6, 2017, using the Wenjuanxing Internet platform (https://www.sojump.com/).

\subsection{Statistical analysis}

IBM SPSS Statistics for Windows, version 20.0, was used for analysis. The nursing time required for medication administration was presented as the mean \pm standard deviation. We applied two independent sample $t$-tests to analyze the difference in nursing time before and after implementation of the closed-loop medication system. The degree of satisfaction of the nurses in the pilot wards was calculated as frequencies and percentages.

\section{Results}

\subsection{Nursing time}

The nursing times required for oral medication administration before and after implementation of the closedloop medication administration system are shown in
Table 1. The average nursing time of the four wards before the new system was $31.56 \pm 10.88$ minutes, which was reduced to $18.74 \pm 5.60$ minutes after the implementation of the system.

\subsection{Nurses' satisfaction}

The nurses' satisfaction with the closed-loop medication administration system is shown in Table 2.

\subsection{MAE alert logs}

There were only $27 \mathrm{MEA}$ alert logs from the repeated scans of 3,428 instances of medication administration during the observation period.

\section{Discussion}

\subsection{The closed-loop medication administration system incorporates several technologies into the workflow of the nursing staff to improve the nurses' work efficiency}

As Table 1 shows, there was a significant difference ( $t=8.85, P<0.00)$ between the two groups. The closedloop system provides an additional layer of safety by real-time scanning of barcodes, which relieves one nurse during medication administration. Medication administration could be performed by a single nurse, who manually checks at the bedside and scans both the barcodes (on patient's wristband and on the medication). ${ }^{18}$ This system can provide nurses more time to focus on the professional steps of medication administration, such as monitoring for adverse events and continuous patient assessment. The system offered a significant improvement in this aspect.

\begin{tabular}{lcc}
\hline \multirow{2}{*}{ Ward } & \multicolumn{2}{c}{ Average nursing time $(\mathrm{M} \pm \mathrm{SD})$, min } \\
\cline { 2 - 3 } & $\begin{array}{c}\text { Before system } \\
\text { adoption }\end{array}$ & $\begin{array}{c}\text { After system } \\
\text { adoption }\end{array}$ \\
\hline \hline Medical Ward 1 & $32.57 \pm 8.72$ & $15.73 \pm 4.57$ \\
Medical Ward 2 & $41.89 \pm 5.44$ & $20.07 \pm 2.25$ \\
Surgical Ward 1 & $29.33 \pm 9.46$ & $18.83 \pm 7.25$ \\
Surgical Ward 2 & $22.70 \pm 9.83$ & $20.75 \pm 6.80$ \\
Total & $31.56 \pm 10.88$ & $18.74 \pm 5.60$ \\
\hline
\end{tabular}

Table 1. Average nursing times for oral medication administration before and after implementation of the system in the pilot wards. Note: Two independent samples $t$-tests
the two groups. $(t=8.85, P=0.00)$ 


\begin{tabular}{|c|c|c|c|c|c|c|c|c|c|c|}
\hline \multirow{2}{*}{ Items } & \multicolumn{2}{|c|}{ Strongly disagree } & \multicolumn{2}{|c|}{ Disagree } & \multicolumn{2}{|c|}{ Neutral } & \multicolumn{2}{|c|}{ Agree } & \multicolumn{2}{|c|}{ Strongly agree } \\
\hline & $n$ & $\%$ & $\mathrm{n}$ & $\%$ & $\mathrm{n}$ & $\%$ & $\mathrm{n}$ & $\%$ & $\mathrm{n}$ & $\%$ \\
\hline $\begin{array}{l}\text { The system can facilitate your work and } \\
\text { reduce your workload }\end{array}$ & 5 & 7.14 & 11 & 15.71 & 21 & 30.00 & 19 & 27.14 & 14 & 20.00 \\
\hline $\begin{array}{l}\text { The system can reduce check time and } \\
\text { enhance work efficiency }\end{array}$ & 3 & 4.29 & 9 & 12.86 & 22 & 31.43 & 19 & 27.14 & 17 & 24.29 \\
\hline $\begin{array}{l}\text { The system can help to improve checking } \\
\text { accuracy and reduce MAEs }\end{array}$ & 2 & 2.86 & 0 & 0 & 10 & 14.29 & 34 & 48.57 & 24 & 34.29 \\
\hline $\begin{array}{l}\text { The system can track MAEs to improve } \\
\text { nursing quality }\end{array}$ & 3 & 4.29 & 6 & 8.57 & 16 & 22.86 & 25 & 35.71 & 20 & 28.57 \\
\hline $\begin{array}{l}\text { The degree of helpfulness of the system } \\
\text { to your work }\end{array}$ & 4 & 5.71 & 1 & 1.43 & 23 & 32.86 & 28 & 40.00 & 14 & 20.00 \\
\hline
\end{tabular}

Table 2. Nurses' satisfaction with the system $(n=70)$.

\subsection{Nurses' satisfaction with the closed-loop medication administration system}

Table 2 shows that $60.00 \%(n=42)$ of nurses considered the system to be helpful for their work and $82.86 \%$ ( $n=58$ ) thought that the system was helpful to improve checking accuracy and reduce MAEs to ensure patient safety. This is a key point for the development and customization of such systems. Developers and implementers must consider nursing workflow in order to make the system easy to access.

\subsection{The closed-loop medication administration system can help nurses achieve safe care}

The MAE alert rate was $1.22 \%$ during the one-year observation period in a previous study, suggesting that the closed-loop medication administration system contributed to improving patient safety by preventing potential MAEs. ${ }^{2}$ In the limited time since the implementation in the present study, there were only 27 MAE alert logs among 3,428 medication administrations during the observation period. The full impact of the closed-loop medication administration system on patient safety will be evaluated and analyzed in future studies.

\section{Conclusions}

Successful implementation and adoption of the closedloop medication administration system are more likely to happen only when the developers and implementers understand the complexities and unpredictability of the nurses' workflow. For instance, an inability to ensure the compatibility of the system with nursing workflow may lead to unintended consequences. Clearer guidance is required from hospitals on the use of the systems by physicians, nurses, and other medical staff. Without clear policies, well-developed systems that are well implemented and designed may hinder nursing workflow and impact patient safety and care. A key policy priority, therefore, is to plan for the long-term use of these systems.

\subsection{Strengths and weaknesses of the study}

This study had three limitations. First, the main weakness of this study is that data were only collected from four pilot wards. Second, the system has only been trialed for half a month, since the open tender process of the PDA was unsuccessful. Third, the project failed to follow the schedule. The MAE alert logs have been evaluated for half a month, which is insufficient for further analysis. The success of the pilot work has laid the foundation for further research and implementation of the system throughout the hospital.

\section{Acknowledgments}

This study was supported by grants from the 2015 Shenzhen Health Bureau Research Project. The system was exploited by project team members and IT engineers from E-well technology Co., Ltd. and funded by HKU-SZH.

We thank all team members, especially Head Nurses CF Liu and JF Cao and the IT Manager Y Lu, for their contributions during the implementation of the system as well as RN FX Xuan and four nursing students for data collection and analysis.

\section{Conflicts of interest}

All contributing authors declare no conflicts of interest. 


\section{References}

1. Jheeta S, Franklin BD. The impact of a hospital electronic prescribing and medication administration system on medication administration safety: An observational study. BMC Health Serv Res. 2017; $17: 547$.

2. Hwang $\mathrm{Y}$, Yoon D, Ahn EK, Hwang H, Park RW. Provider risk factors for medication administration error alerts: Analyses of a large-scale closedloop medication administration system using RFID and barcode. Pharmacoepidemiol Drug Saf. 2016;25:1387-1396.

3. Zhang $Y$, Long $X$, Chen W, Li H, Duan H, Shang $Q$. A concise drug alerting rule set for Chinese hospitals and its application in computerized physician order entry (CPOE). Springerplus. 2016;5:2067.

4. Romanow D, Rai A, Keil M, Luxenberg S. Does extended CPOE use reduce patient length of stay? Int J Med Inform. 2017;97:128-138.

5. Rodriguez Torres $\mathrm{Y}$, Huang J, Mihlstin M, Juzych MS, Kromrei H, Hwang FS. The effect of electronic health record software design on resident documentation and compliance with evidence-based medicine. PLoS One. 2017;12:e0185052.

6. Coaker H. Electrically controlled drug-delivery system may help minimize side effects: Remotely controlled drug-delivery device may be a step towards 'smart' closed-loop drug-eluting systems. Nanomedicine (Lond). 2014;9:569-570.

7. Furukawa MF, Raghu TS, Shao BB. Electronic medical records, nurse staffing, and nurse-sensitive patient outcomes: Evidence from California hospitals, 1998-2007. Health Serv Res. 2010;45: 941-962.

8. Zhang LM, Yu YY, Liu YP. The close-loop system and medication safety. Chin Health Quality Manag. 2016;3:7-9 (in Chinese).
9. Pan HY, Zheng SW, Zhong FF, et al. Development and application of a closed-loop medication orders executive system. Chin J Nurs. 2012;9:818-820 (in Chinese).

10. Institute of Medicine. To Err is Human: Building A Safer Health System. Washington, DC: National Academy Press; 1999.

11. Agrawal A. Medication errors: Prevention using information technology systems. Br J Clin Pharmacol. 2009;67:681-686.

12. Franklin BD, O'Grady K, Donyai $P$, Jacklin A, Barber $\mathrm{N}$. The impact of a closed-loop electronic prescribing and administration system on prescribing errors, administration errors and staff time: A before-andafter study. Qual Saf Health Care. 2007;16:279-284.

13. Ma ZL, Zhang L. Role of HIS in the modernization efforts of hospitals. Chin J Hosp Admin. 2006;5:350351 (in Chinese).

14. Househ M, Ahmad A, Alshaikh A, Alsuweed F. Patient safety perspectives: The impact of $\mathrm{CPOE}$ on nursing workflow. Stud Health Technol Inform. 2013;183:367-371.

15. Wu P, Zhang JW. Closed-loop management model of clinical investigational product for new drug of traditional Chinese medicine. China J Chin Materia Medica. 2013;38:2898-2900 (in Chinese).

16. Bonkowski J, Carnes C, Melucci J, et al. Effect of barcode-assisted medication administration on emergency department medication errors. Acad Emerg Med. 2013;20:801-806.

17. Chen MY, Zheng KL, Man W. Application of the closed loop medication system. Chin J Hosp Admin. 2012;11:858-859 (in Chinese).

18. Poon EG, Keohane CA, Yoon CS, et al. Effect of bar-code technology on the safety of medication administration. N Engl J Med. 2010;362:1698-1707.

How to cite this article: Shi LP, Liu CH, Cao JF, et al. Development and application of a closed-loop medication administration system in University of Hongkong-Shenzhen Hospital. Front Nurs. 2018; 2: 105-110. https://doi. org/10.2478/fon-2018-0014 